\title{
Serum neopterin concentrations in chronic liver disease
}

\author{
A Wilmer, B Nölchen, H Tilg, M Herold, Ch Pechlaner, G Judmaier, O Dietze, W Vogel
}

\begin{abstract}
Neopterin is produced by macrophages after stimulation with interferon $\gamma$ or lipopolysaccharide. Its production is increased in many infectious, autoimmune, and malignant diseases. The aim of this study was to examine whether, on the basis of neopterin as a marker, liver diseases could be classified according to aetiology and stage of disease. A cohort of 264 patients with chronic liver diseases (viral, metabolic, autoimmune, toxic) and 150 normal controls were studied; 136 of the patients had cirrhosis. Increased serum neopterin concentrations were found in $41 \%$ of all patients (controls 6.0 $(2 \cdot 2) \mathrm{nmol} / \mathrm{l})$, with patients in the cirrhotic stage of disease showing higher neopterin values (mean (SD) $15.7(23.6) \mathrm{nmol} / \mathrm{ml}$ ) than those in the non-cirrhotic stage $\mathbf{( 9 . 9}$ $(5 \cdot 5))$. There were no statistically significant differences in the serum neopterin concentrations that could be considered characteristic for different stages of disease classified according to the Child criteria. Such differences in concentrations of neopterin that were found in patients with liver diseases grouped according to underlying causes were only marginal. Serum neopterin concentrations were found to be significantly lower than in any other disease group only in patients with Wilson's disease. The results suggest that activated macrophages participate in the development of chronic liver disease. Measurement of serum neopterin does not offer a reliable method for differentiating between various aetiologies of chronic liver diseases and does not help to predict severity of cirrhosis.
\end{abstract}

(Gut 1995; 37: 108-112)

Keywords: neopterin, chronic liver disease.

A Wilmer

B Nölchen

$\mathrm{H}$ Tilg

$M$ Herold

Ch Pechlaner

G Judmaier

W Vogel

and Pathology

O Dietze

School of Medicine, University of

Innsbruck,

Innsbruck, Austria

Correspondence to:

Dr W Vogel, Department of Internal Medicine, Innsbruck University, Anichstr 35, A-6020 Innsbruck, Austria.

Accepted for publication 10 November 1994

Interferon $\gamma$, which is released from activated $T$ cells, and lipopolysaccharide trigger human macrophages to produce and secrete neopterin. ${ }^{1-3}$ Neopterin belongs to the family of pteridines and is a degradation product arising from the biosynthesis of tetrahydrobiopterin. ${ }^{45}$ Its structure (2-amino-4-hydroxy-6-[1,2,3,trihydroxy-propyl]-pteridine) was identified by Rembold and Buschmann in $1963 .{ }^{6}$ As no other cell type is known to produce this substance, neopterin, with no immunological function of its own, is an indicator for the activation of the cellular immune system, for endogenous macrophage activation. ${ }^{57} \mathrm{~A}$ positive correlation between immune response and increased concentrations of neopterin has been shown in vivo and in vitro. ${ }^{2}$ Several clinical studies have shown increased neopterin concentrations in serum or urine, or both, of patients with diseases known to be associated with an activation of the cellular immune system such as viral (for example, viral hepatitis ${ }^{8-10}$ ), and bacterial infections, ${ }^{11} 12$ allograft rejection, ${ }^{13} 14$ graft versus host disease, ${ }^{15}$ and autoimmune diseases. ${ }^{716}$ Thus, neopterin can be regarded as reflecting the clinical activity of these diseases. ${ }^{716}$ Furthermore, increased neopterin concentrations were found not only in various malignant diseases ${ }^{5}$ but also in severe infectious diseases such as HIV. In all these cases, enhanced concentrations of neopterin have been shown to have prognostic significance. ${ }^{57}$

Chronic liver diseases of different aetiologies may all lead to cirrhosis. ${ }^{1718}$ The pathophysiology of such chronic inflammatory processes is poorly understood. A number of studies, however, have suggested that changes in regulatory $T$ cell function and thus abnormal regulation of the immune response are to be considered as important factors. ${ }^{19}$ The role of the macrophage as mediator/regulator of $\mathrm{T}$ cell activity and the extent of its contribution in the development of fibrosis remain to be elucidated.

The aim of this study was to examine whether neopterin can be used as a marker to determine differences in liver diseases according to aetiology and stage of disease, and whether neopterin concentrations could be correlated with other laboratory and clinical parameters. We therefore studied serum neopterin concentrations in 264 patients representing a broad aetiological spectrum of cirrhotic and non-cirrhotic liver diseases.

\section{Methods} interferon $\gamma$ production, and for monocyte/

\section{Patients}

A total of 264 patients (136 cirrhotic, 128 noncirrhotic) with chronic liver disease and 150 normal controls were studied. Mean (SD) age of the 62 women and 88 men was 47 (12) years. Diagnosis of chronic liver disease was confirmed by liver biopsy in all non-cirrhotic patients. Diagnosis of cirrhosis was established by biopsy or on the basis of the presence of physical stigmata of chronic liver disease such as cutaneous liver signs, hepatosplenomegaly, gynecomastia, testicular atrophy, muscle wasting, together with biochemical evidence of impaired hepatic synthetic function (albumin, 
prothrombin time). The stage of cirrhosis was classified according to the Child-Pugh criteria: 82 patients were in Child A, 33 in Child $B$, and 21 in Child $C$ stage. None of them had active variceal bleeding at the time of investigation. Patients with fever, HIV infection, acute hepatic disease, overt infectious diseases such as septicaemia, pneumonia or other extrahepatic infectious disease, renal insufficiency or those receiving immunodulatory therapy were excluded from the study.

Chronic hepatitis $B$ virus infection ( $H B V$ infection) - this patient group comprised 37 subjects seropositive for hepatitis B surface and e-antigen for at least six months. The histological diagnoses were: chronic persistent hepatitis $(n=4)$, chronic active hepatitis $(n=13)$, and cirrhosis $(n=20)$. All patients were negative for delta antibody.

Chronic hepatitis $C$ virus infection (HCV infection) - 52 patients fulfilled the diagnostic criteria of chronic hepatitis C. Patients were entered into this group if they had biopsy findings consistent with chronic hepatitis C and were repeatedly shown to be positive for antibodies to HCV. Thirty one patients had chronic active hepatitis and 21 had cirrhosis.

Primary biliary cirrhosis - the criteria for establishing the diagnosis were the presence of disease specific anti-mitochondrial antibodies of the M2 subtype (Pharmacia ELISA) together with increased serum concentrations of alkaline phosphatase and immunoglobulin $M$ (Ig-M) or histopathological findings consistent with primary biliary cirrhosis diagnosis $(n=40)$, or both. This group included 46 patients, 11 of whom were in the cirrhotic stage.

Autoimmune liver disease - all 15 patients of this group fulfilled the generally accepted criteria for diagnosis of autoimmune chronic active hepatitis ${ }^{20}$ namely, presence of antinuclear or antismooth muscle autoantibodies $(>1: 40)$, or both, hypergammaglobulinaemia (mainly IgG), and liver biopsy specimens exhibiting both chronic inflammatory changes and hepatocellular necrosis. All patients had been in clinical and biochemical remission for at least six months during which they received no immunosuppressive treatment before being included in the study with relapsing disease. Cirrhosis was seen in nine of 15 patients.

Metabolic liver disease (Wilson's disease, genetic haemochromatosis, $\alpha 1$ antitrypsin deficiency), were diagnosed in 39 patients. Diagnoses were established according to accepted standard criteria. Nine patients presented with Wilson's disease; haemochromatosis was diagnosed in 14, and $\alpha 1$ antitrypsin deficiency in 16 patients, two of whom showed the $\mathrm{ZZ}$ and 14 the $\mathrm{MZ}$ phenotype. ${ }^{21}$ Patients with Wilson's disease and genetic haemochromatosis were receiving active treatment during the study with d-penicillamine or phlebotomy. All patients studied had increased body stores of iron and copper respectively as determined by urinary copper excretion or serum ferritin.

Alcoholic liver disease - this group included 30 patients. Seven of these presented no symptoms of cirrhosis and 23 with stable alcoholic cirrhosis. Diagnosis was established on the basis of exclusion of other liver diseases, history of alcohol consumption of at least $80 \mathrm{~g}$ ethanol daily for several years, and histological findings $(n=21)$. Diagnosis of alcohol abuse was further based on psychiatric examination. All patients considered themselves as abstaining from alcohol when they attended our outpatients department.

Primary hepatocellular carcinoma - diagnosis was confirmed by histological findings or fine needle aspiration cytology in nine of 17 cases. Computed tomography and extensive increases in serum $\alpha$ fetoprotein concentrations established diagnosis in the rest of the patients of this group. Fifteen patients with hepatocellular carcinoma were cirrhotic, the underlying diseases being chronic hepatitis B infection $(n=1)$, chronic hepatitis $C$ infection $(n=8)$, haemochromatosis $(n=3)$, alcoholic liver disease $(n=4)$, and $\alpha 1$ antitrypsin deficiency $(\mathrm{n}=1)$.

Multifactorial liver disease - 28 patients, of whom 22 were cirrhotic, had a combination of two or more different causes of liver disease. In 21 patients there was a combination of $\mathrm{HCV}$ infection with either alcoholic liver disease $(n=11), H B V$ infection $(n=4), \alpha 1$ antitrypsin deficiency $(n=4)$, autoimmune liver disease $(n=1)$, or Wilson's disease $(n=1)$. In seven patients, alcoholic liver disease was combined with $\alpha 1$ antitrypsin deficiency $(n=4)$, haemochromatosis $(n=1)$, or primary biliary cirrhosis $(n=2)$.

\section{Study design}

Viral markers - commercially available radioimmunoassays were used to detect hepatitis B surface and e antigens and antihepatitis delta antibodies (Abbott Laboratory). Antibodies to $\mathrm{HCV}$ were detected using a commercially available enzyme immunoassay (second generation HCV-ELISA, Abbot diagnostics). In addition, all serum samples were tested for anti-HIV antibodies (Abbott System).

Liver biopsies - biopsy specimens were graded for their degree of periportal, portal, and lobular inflammation and fibrosis, according to the scoring system proposed by Knodell et al. ${ }^{22}$

Blood collection - venous blood was collected into sterile endotoxin free vacuum blood collection tubes and allowed to clot at room temperature for 30 minutes. Until use serum was stored at $-20^{\circ} \mathrm{C}$. Analyses were performed after the first thawing and within six months of storage.

Neopterin in serum was measured by a commercially available radioimmunoassay (Neopterin-RIAcid, Henning Berlin $\mathrm{GmbH}$, Berlin, Germany), which is based on a double antibody technique using a pre-precipitated antiserum and ${ }^{125} \mathrm{I}$-labelled neopterin as tracer. Samples were centrifuged at $700 \times g$ and protected from light to prevent degradation of neopterin. Serum values in a cohort of 150 normal controls were $6 \cdot 0(2 \cdot 2)$ (mean (SD)). 
Neopterin serum concentrations (nmoll) in various chronic liver disease (mean (SEM))

\begin{tabular}{|c|c|c|c|c|c|c|}
\hline \multirow[b]{2}{*}{ Diagnosis* } & \multicolumn{2}{|c|}{ All patients } & \multicolumn{2}{|c|}{ Non-cirrhotic patients } & \multicolumn{2}{|c|}{ Cirrhotic patients } \\
\hline & Number & $\begin{array}{l}\text { Serum } \\
\text { concentrations }\end{array}$ & Number & $\begin{array}{l}\text { Serum } \\
\text { concentrations }\end{array}$ & Number & $\begin{array}{l}\text { Serum } \\
\text { concentrations }\end{array}$ \\
\hline $\begin{array}{l}\text { HBV } \\
\text { HCV } \\
\text { GH } \\
\text { WD } \\
\text { A1AT } \\
\text { PBC } \\
\text { AUTO } \\
\text { ALD } \\
\text { HCC } \\
\text { MLD }\end{array}$ & $\begin{array}{l}(\mathrm{n}=37) \\
(\mathrm{n}=52) \\
(\mathrm{n}=14) \\
(\mathrm{n}=9) \\
(\mathrm{n}=16) \\
(\mathrm{n}=46) \\
(\mathrm{n}=15) \\
(\mathrm{n}=30) \\
(\mathrm{n}=17) \\
(\mathrm{n}=28)\end{array}$ & $\begin{array}{r}9.7(1.3) \\
12.3(0.9) \\
9.0(1 \cdot 7) \\
5.4(0.8) \\
14 \cdot 1(3.9) \\
11.0(1.0) \\
11 \cdot 1(1.2) \\
14.8(5.1) \\
15.5(3.0) \\
22.1(7.8)\end{array}$ & $\begin{array}{l}(n=17) \\
(n=31) \\
(n=8) \\
(n=6) \\
(n=10) \\
(n=35) \\
(n=6) \\
(n=7) \\
(n=2) \\
(n=6)\end{array}$ & $\begin{array}{r}8.1(1.4) \\
11.1(1.0) \\
7.8(1.0) \\
6.3(1.0) \\
8.3(1.4) \\
11.0(1.1) \\
10.2(2.4) \\
11.4(3.0) \\
13.0(5.8) \\
8.8(1.4)\end{array}$ & $\begin{array}{l}(n=20) \\
(n=21) \\
(n=6) \\
(n=3) \\
(n=6) \\
(n=11) \\
(n=9) \\
(n=23) \\
(n=15) \\
(n=22)\end{array}$ & $\begin{array}{r}11.2(2.0) \\
14.7(1.7) \\
11.0(4.3) \\
3.6(0.6) \\
23.8(9.1) \\
11.1(2.9) \\
11.7(1.4) \\
15.5(6.2) \\
15.8(3.4) \\
25.1(9.4)\end{array}$ \\
\hline All & $(n=264)$ & $12 \cdot 9(1 \cdot 1)$ & $(n=128)$ & $9.9(0.5)$ & $(n=136)$ & $15 \cdot 7(2 \cdot 1)$ \\
\hline
\end{tabular}

(HBV=hepatitis B, HCV=hepatitis C, GH=genetic haemochromatosis, WD=Wilson's disease, $\mathrm{A} 1 \mathrm{AT}=\alpha 1$ antitrypsin deficiency, $\mathrm{PBC}=$ primary biliary cirrhosis, AUTO=autoimmune hepatitis, $\mathrm{ALD}=$ alcoholic liver disease, $\mathrm{HCC}=$ primary hepatocellular carcinoma,

$M L D=$ multifactorial liver disease).

Biochemical investigations - all patients received the same routine biochemical investigations alanine aminotransferase, aspartate aminotransferase, total bilirubin, alkaline phosphatase, $\gamma$-glutamyltransferase, total protein, albumin, serum immunoglobulin concentrations (IgG, IgM, IgA), creatinine, cholesterol, triglyceride, transferrin, ferritin, serum iron (tissue iron for the diagnosis of haemochromatosis), serum and urine copper (tissue copper if necessary for the diagnosis of Wilson's disease), caeruloplasmin, prothrombin time, $\alpha$ antitrypsin phenotype (isoelectric focusing), erythrocytes, haemoglobin, packed cell volume, leucocytes, thrombocytes, and erythrocyte sedimentation rate.

\section{Statistical analysis}

Neopterin values were not normally distributed, but were so after log transformation. Differences between groups were then tested with Student's $t$ test. Results are given as mean (SD) or (SEM), when appropriate.

Log transformed neopterin values were also used in multivariate analysis. The assumption of equal variances between groups had to be rejected at the 0.05 values, as shown by Levene's test. Accordingly, the BrownForsythe and Welch procedures were applied to test for equality of means between groups of different aetiology and Child stage. Differing groups were identified by pairwise comparison using Student's $t$ test. Because of the explorative design of our study, we report not only the results obtained after correction for multiple significance testing (Bonferroni method), but also differences before correction, so as not to miss results of possible significance or interest. Correlations were analysed with Pearson's coefficient. All calculations were performed by use of the BMPD statistical software package (Los Angeles, California).

\section{Results}

Increased serum neopterin concentration $(>10 \mathrm{nmol} / \mathrm{l})$ was found in $41 \%$ of all patients $(n=264)$. Mean serum neopterin concentrations were $12.9 \mathrm{nmol} / 1$ in patients (SEM $1 \cdot 1$, range $3 \cdot 0-183 \cdot 7$ ) and $6.0 \mathrm{nmol} / 1$ in normal controls (SEM 2.2, range 2.4-12.7). Neopterin concentrations scattered over a wide range ( $<3.0$ to $183.7 \mathrm{nmol} / \mathrm{l})$, with a long right sided tail (median 8.9).

\section{Neopterin in cirrhotic and non-cirrhotic chronic} liver disease

Serum neopterin in patients with cirrhosis $(n=136)$ was higher when compared with noncirrhotic patients (mean (SD): $15.7(23.6) v$ $9.9(5.5) \mathrm{nmol} / \mathrm{l}, 95 \%$ confidence intervals 11.6 to $19.7 \vee 8.9$ to $10.8 ; \mathrm{p}=0.03$ ). When examining each aetiologic group separately (Table), neopterin tended to be higher in patients with cirrhosis, with the exception of Wilson's disease $(6.2 v 3.6 \mathrm{nmol} / \mathrm{l} ; \mathrm{p}=0.06)$. The difference was significant only in patients with $\alpha 1$ antitrypsin deficiency (8.3 $v 23.7$ $\mathrm{nmol} / \mathrm{l}, \mathrm{p}=0.005)$.

In addition, cirrhotic patients were analysed according to Child classification. Neopterin concentrations were highest in Child B patients $(n=32$; mean (SD): $22 \cdot 7(33.8)$ ), but were very similar in Child A $(n=83 ; 13.6(20.6))$ and $C$ patients $(n=18 ; 13.0(9.0))$. These differences were not statistically significant when tested by multivariate analysis.

\section{Neopterin in non-cirrhotic liver disease related to} aetiology

The Table and Figure show serum neopterin concentrations in various underlying noncirrhotic liver diseases. Neopterin values scattered over a range of 3.0 to $34.2 \mathrm{nmol} / \mathrm{l}$, concentrations above 30.0 were seen in primary biliary cirrhosis and HBV. Multivariate analysis showed no significant differences between group means when correcting for multiple significance testing. However, before applying correction according to Bonferroni, neopterin values in patients with Wilson's disease were lower than in primary biliary cirrhosis and HCV patients at the 0.05 value, as was neopterin in $\mathrm{HBV}$ patients when compared with $\mathrm{HCV}$ or primary biliary cirrhosis patients.

\section{Neopterin in cirrhotic liver disease related to aetiology}

The Table and Figure show the neopterin concentrations related to different aetiologies. Values varied over a range of $<3.0$ to 183.7 $\mathrm{nmol} / \mathrm{l}$, the highest individual values were seen in patients with multifactorial liver disease (183.7), alcoholic liver disease (146.7), $\alpha 1$ antitrypsin deficiency $(68 \cdot 8)$, and hepatocellular carcinoma (57-2). Again, patients with Wilson's disease had appreciably low neopterin values. Multivariate analysis disclosed no significant differences in group means. Pairwise comparison before correcting for multiple significance testing, however, showed neopterin in patients with Wilson's disease to be lower than in patients with HCV $(p=0.01)$, alcoholic liver disease $(p=0.05)$, autoimmune liver disease $(p=0.03), \alpha 1$ antitrypsin deficiency $(p=0.003)$, and hepatocellular carcinoma $(p=0.01)$. 


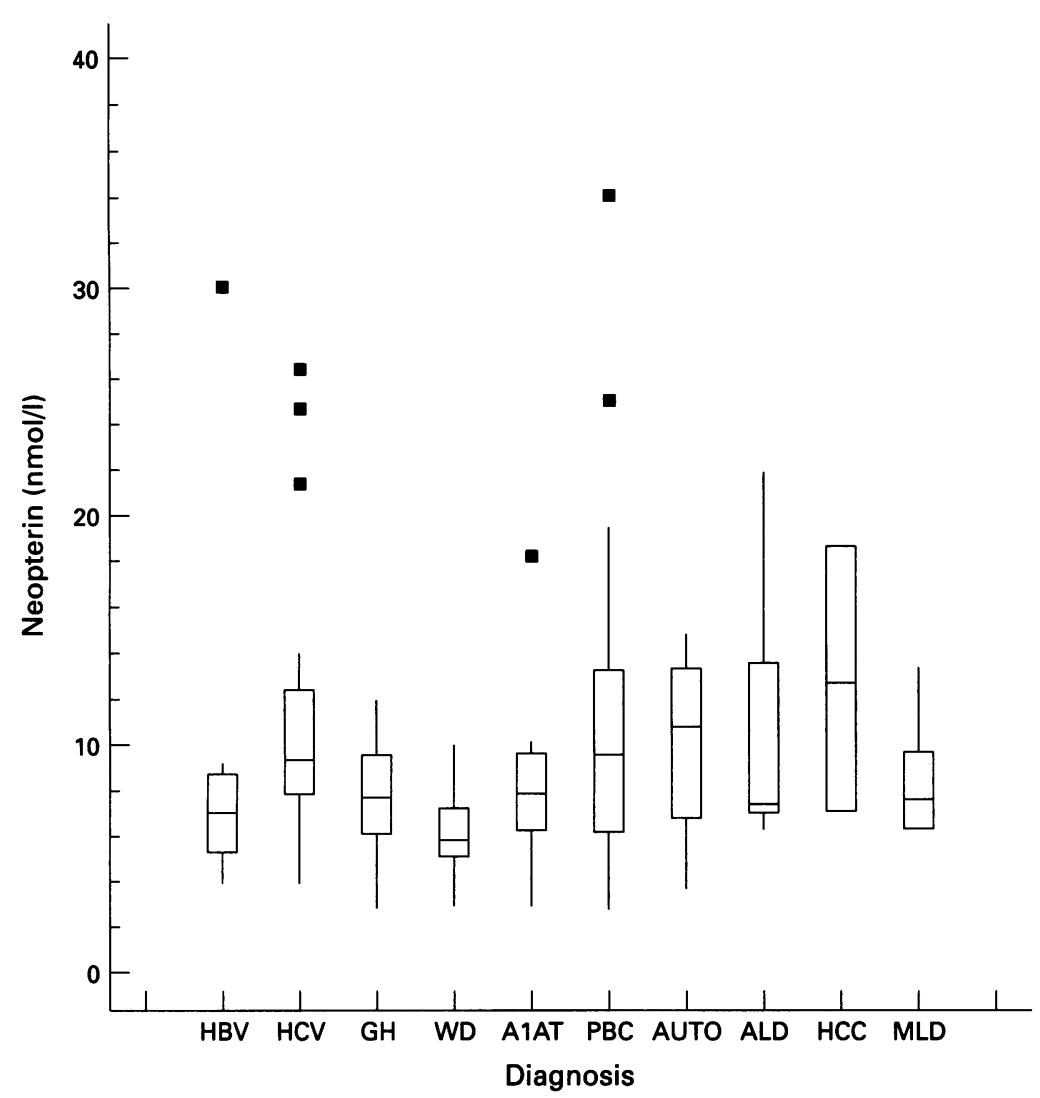

Serum concentrations of neopterin in non-cirrhotic patients $(n=128)$ with various chronic liver diseases. Values are shown in box and whisker plots: bars include median, $25 \%$ and $75 \%$ percentiles; lines show $95 \%$ percentiles. Abbreviations as shown in Table.

Neopterin in chronic liver disease independent of stage of disease related to aetiology

The analysis of neopterin concentrations of all patients according to the aetiology of disease alone, irrespective of the stage, gave the same results as the analysis related to both stage and aetiology. The Table gives the details. By far the lowest neopterin serum concentrations were found in patients with Wilson's disease. Multivariate analysis proved these patients to have lower mean neopterin $(5 \cdot 4(2 \cdot 3) \mathrm{nmol} / \mathrm{l})$ than patients with $\mathrm{HCV}$ $(12 \cdot 3(6 \cdot 7))$ and patients with hepatocellular carcinoma $(15.5(12.5))$, at the 0.05 value after Bonferroni correction. On pairwise testing, before Bonferroni, neopterin concentrations in Wilson's disease patients were also lower than in patients with primary biliary cirrhosis $(p=0.007)$, alcoholic liver disease $(p=0.01)$, autoimmune liver disease $(p=0.01), \alpha 1$ antitrypsin deficiency $(p=$ $0.006)$, multifactorial liver disease $(p=$ $0.002)$, and HBV $(p=0.04)$. The difference in mean neopterin was not significant only when compared with haemochromatosis patients.

Comparison of neopterin concentrations with other diagnostic parameters

Most of the diagnostic parameters of liver cell injury (transaminases, bilirubin), serum immunoglobulin concentrations, alkaline phosphatase, total protein, creatinine, cholesterol, triglyceride, transferrin, ferritin, and serum iron did not correlate with serum neopterin concentrations. There were only weak negative correlations with albumin $(r=-0.14 ; \quad \mathrm{p}<0.03)$, prothrombin time $(r=-0.18 ; \mathrm{p}<0.005)$, haemoglobin $(r=-0.29$; $\mathrm{p}<0.00001)$, packed cell volume $(r=-0.28$; $\mathrm{p}<0.00001), \quad$ erythrocytes $\quad(r=-0.22$; $\mathrm{p}<0.001)$, leucocytes $(r=-0.16 ; \mathrm{p}<0.014)$, thrombocytes $(r=-0.14 ; \mathrm{p}<0.043)$, and a similar weak but positive correlation with erythrocyte sedimentation rate $(r=0.23$; $\mathrm{p}<0.05)$.

\section{Discussion}

Neopterin is a marker for both macrophage activity and cell mediated immunity. ${ }^{57}$ Immunopathology of chronic liver disease is as yet poorly understood, although various abnormal findings have been described in both the humoral and the cellular limb of the immune system. ${ }^{19}$ In this study, the serum concentration of neopterin was determined in patients with the whole spectrum of chronic liver diseases in the cirrhotic as well as the noncirrhotic stage. Increased neopterin concentrations have been found in $41 \%$ of all patients with chronic liver disease irrespective of underlying cause and stage of disease. Patients with cirrhosis had distinctly higher serum neopterin concentrations than those in the non-cirrhotic stage of disease, however, there were no statistically significant differences in the values of neopterin as far as the three stages are concerned.

Chronic liver diseases are heterogeneous in their aetiology, but in all cases, cirrhosis remains a possible end stage. Cirrhosis is mainly characterised by an increase of fibrous tissue and a compensatory proliferation of hepatocytes in an irregular manner resulting from necroinflammation. Although the mechanisms of parenchymal regeneration are not well understood, involvement of cytokines is generally assumed.181923 Activated macrophages secrete soluble mediators such as interleukin 1, tumour necrosis factor $\alpha$, platelet derived growth factor, and transforming growth factor $\beta$, all of which are fibrogenetic. ${ }^{24}$ Indeed, independent of aetiology, patients in the cirrhotic stage had higher concentrations of serum neopterin, which is secreted exclusively by activated macrophages. As the clearance capacity of the liver for a wide range of peptides is reduced in the cirrhotic stage, it is conceivable that a higher level of substances known to stimulate macrophages (for example, immune complexes, endotoxin) are circulating in the bloodstream, which might explain the higher neopterin concentrations in cirrhotic patients.

The differences in the increased neopterin concentrations related to the various aetiologies investigated were marginal. Only patients with Wilson's disease showed normal neopterin values. These values were significantly lower than the concentrations measured in any other group. This finding suggests that in the pathogenesis of hepatic copper toxicity activation of macrophages is of little importance. As none of the patients studied showed histological evidence of chronic active hepatitis 
it can be assumed that copper deposition induces hepatic lesions by other means than by activation of the full inflammatory cascade, which would cause increased production of neopterin. The other explanation that treatment with d-penicillamine would directly suppress neopterin production has to remain speculative. Interestingly, $\alpha 1$ antitrypsin deficiency compared with the other storage diseases examined showed surprisingly high serum neopterin concentrations. The highly significant differences between cirrhotic and non-cirrhotic cases of $\alpha 1$ antitrypsin deficiency were also considerable. Neopterin values in the cirrhotic stage of $\alpha 1$ antitrypsin deficiency were nearly three times higher than in the noncirrhotic stage. The reason for this striking rise in the neopterin concentrations is not clear. It may reflect a specific dysregulation of the whole inflammatory process in which $\alpha 1$ antitrypsin is a regulatory protein.

Furthermore, we found increased neopterin concentrations in autoimmune hepatitis and primary biliary cirrhosis, confirming the findings in other autoimmune disorders. Patients with hepatocellular carcinoma showed the highest neopterin values in our study (with the exception of multifactorial liver diseases). In various malignant disorders enhanced neopterin values were reported, but it is as yet unclear, whether the tumour itself produced neopterin or whether it is the immune system that is responsible for increased neopterin synthesis.

There are only a few studies on the role of neopterin as a marker in liver disease. In two previous studies, increased urinary neopterin concentrations were reported in both acute and chronic viral hepatitis. ${ }^{89}$ Ninety per cent of the patients in the first group and $88.6 \%$ of those in the second group had increased neopterin concentrations compared with only $6 \%$ of patients who presumably had alcoholic liver disease. ${ }^{89}$ It was suggested that measurement of neopterin could help differentiate between viral and alcoholic liver diseases. ${ }^{89}$ Based on these studies it was recently proposed that serum neopterin could be reliably used as a marker for virus free blood products. ${ }^{23}$ Our study, however, shows that there are no significant differences between the values of neopterin in patients with chronic viral hepatitis and those with alcoholic liver disease; and there are patients with chronic viral hepatitis with normal serum neopterin values. These results are in contrast with the two previous studies, in which urinary neopterin concentrations of rather small patient groups were compared. Also, with these results, we found no strong correlation (correlation coefficient $r<0.5^{25}$ ) between concentrations of serum neopterin and other biochemical parameters.

In a smaller Japanese study ${ }^{26}$ comparing viral and alcoholic liver disease, patients with viral hepatitis had higher neopterin concentrations. There was a substantial number of patients, however, with chronic hepatitis producing normal values of neopterin and no patients with metabolic liver diseases were studied.
In conclusion, our results suggest that activated macrophages participate in chronic liver diseases progressing to cirrhosis. Furthermore, the claim that neopterin could be used as a specific marker to differentiate various aetiologies of chronic liver diseases cannot be substantiated.

The authors are grateful to Miss G Schönhuber for excellent technical assistance.

This work is part of the MD thesis by A Wilmer.

1 Huber C, Batchelor JR, Fuchs D, Hausen A, Lang A, Niederwieser $\mathrm{D}$, et al. Immune response-associated production of neopterin. $\mathcal{F}$ Exp Med 1984; 160: 310-6.

2 Woloszczuk W, Troppmair W, Leiter E, Flener R, Schwarz $\mathrm{M}$, Kovarik J, et al. Relationship of interferon-gamma and neopterin levels during stimulation with alloantigens in vivo and in vitro. Transplantation 1986; 41: 716-9.

3 Troppmair J, Nachbaur K, Herold M, Aulitzky WE, Tilg H, Gastl G, et al. In-vitro and in-vivo studies on the induction of neopterin in biosynthesis of cytokines, alloantigens and of neopterin in biosynthesis of cytokines, alloantigens and lipopolysa

4 Blau N, Schoedon G, Curtius H-C. Biosynthesis and significance of neopterin in the immune system. Eur $\mathcal{F}$ Cancer Clin Oncol 1989; 25: 603-5.

5 Huber C, Troppmair J, Rokos H, Curtius HC. Neopterin today. Dtsch Med Wochenschr 1987; 112: 107-13.

6 Rembold H, Buschmann L. Structure and synthesis of neopterin. Liebigs Ann Chem 1963; 662: 72-82.

7 Watcher H, Fuchs D, Hausen A, Reibnegger G, Wetner ER. Neopterin as marker for activation of cellular immunity: immunologic basis and clinical application. Adv Clin Chem 1989; 27: 82-125.

8 Farci AMG, Laconi R, Cabras F. Urinary neopterin in acute and chronic liver disease. In: Wachter $\mathrm{H}$, Curtius HC, Pfeiderer W, eds. Biochemical and clinical aspects of Pfeiderer W, eds. Biochemical and clinical

9 Prior C, Fuchs D, Hausen A, Judmaier G, Reibnegger G, Werner ER, et al. Potential of urinary neopterin excretion in differentiating chronic non-A, non-B hepatitis from fatty liver. Lancet 1987; ii: 1235-7.

10 Reibnegger G, Auhuber I, Fuchs D, Hausen A, Judmaier G, Prior C, et al. Urinary neopterin levels in acute viral hepatitis. Hepatology 1988; 8: 771-4.

11 Denz H, Fuchs D, Hausen A, Huber H, Nachbaur D, Reibnegger $G$, et al. Value of urinary neopterin in the differential diagnosis of bacterial and viral infections. Klin Wochenschr 1990; 68: 218-22.

12 Wachter $\mathrm{H}$, Hausen A, Grassmayr K. Increased urinary excretion of neopterin in patients with malignant tumors and virus diseases. Hoppe Seylers $Z$ Physiol Chem 1979; 360: $1957-60$.

13 Margreiter R, Fuchs D, Hausen A, Huber C, Reibnegger G, Spielberger $M$, Wachter $H$. Neopterin as a new biochemical marker for diagnosis of allograft rejection. Transplantation 1983; 36: 650-3.

14 Tilg H, Vogel W, Aulitzky WE, Schönitzer D, Margreiter R, Deitze $O$, et al. Neopterin excretion after liver transplantation and its value in differential diagnosis of complications. Transplantation 1989; 48: 594-9.

15 Niederwieser D, Huber Ch, Gratwohl A, Bannert P, Fuchs $\mathrm{D}$, Hausen $\mathrm{A}$, et al. Neopterin as a new biochemical marker in the clinical monitoring of bone marrow transplant recipients. Transplantation 1984; 38: 497-500.

16 Hausen A, Fuchs D, Reibnegger G, Wachter H, Egg D, Günther R. Neopterin as index for activity of disease in patients with rheumatoid artiritis. In: Curtius HC, Pfleiderer W, Wachter $\mathrm{H}$, eds. Biochemical and clinical aspects of pteridines. Berlin: De Gruyter, 1983: 245-54.

17 Andus T, Bauer J, Gerok W. Effects of cytokines on the liver. Hepatology 1991; 13: 364-75.

18 Bissel DM, Roll J. Connective tissue metabolism and hepatic fibrosis. In: Zakim D, Boyer TD, eds. Hepatology. 2nd ed. Philadelphia: WB Saunders, 1990: 424-31.

19 Thomas HC. Immunological mechanisms in chronic liver disease. In: Zakim D, Boyer TD, eds. Hepatology. 2nd ed. Philadelphia: WB Saunders, 1990: 1114-26.

20 De Groote J, Desmet VJ, Gegegk P, Korb G, Popper H, Poulsen $\mathrm{H}$, et al. A classification of chronic hepatitis. Poulsen H, et al. A clas 1969 ; ii: $626-8$.

21 Carrel RW, Jeppson JO, Laurel CB. Structure and variation of human alpha- ${ }_{-1}$ antitrypsin. Nature 1982; 298: 329-33.

22 Knodell RG, Ishak KG, Black WC, Chen TS, Craig R, Kaplowitz N, et al. Formulation and application of a numerical scoring system for assessing histological activity in asymtomatic chronic active hepatitis. Hepatology 1981; 1: 431-5

23 Hönlinger M, Fuchs D, Hausen A, Reibnegger G, Schönitzer $\mathrm{D}$, Werner Er, et al. Serum-Neopterinbestimmung zur zusätzlichen Sicherung der Bluttransfusion. Dtsch Med Worchenschr 1989; 114: 172-6.

24 Kovacs EJ. Fibrogenetic cytokines: the role of immune mediators in the development of scar tissue. Immunol Today 1991; 12: 17-23.

25 Immich H. Medical statistics. 1 st ed. Stuttgart: FK Schattauer, 1974: 432-4.

26 Daito K, Suou T, Kawasaki H. Clinical significance of serum and urinary neopterin levels in patients with various liver diseases. Am 7 Gastroenterol 1992; 87: 471-6. 